\title{
Prevention of sudden cardiac death in children and young adults
}

\author{
Aro, Aapo L.
}

2017-06

Aro , A L \& Chugh , S S 2017 , ' Prevention of sudden cardiac death in children and young adults ' , Progress in Pediatric Cardiology, vol. 45 , pp. 37-42 . https://doi.org/10.1016/j.ppedcard.2017.03.003

http://hdl.handle.net/10138/299285

https://doi.org/10.1016/j.ppedcard.2017.03.003

publishedVersion

Downloaded from Helda, University of Helsinki institutional repository.

This is an electronic reprint of the original article.

This reprint may differ from the original in pagination and typographic detail.

Please cite the original version. 


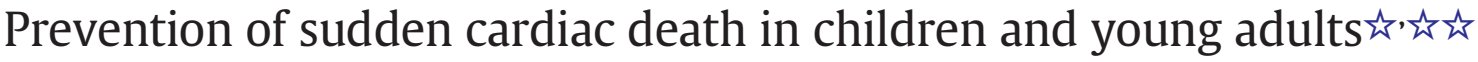

\author{
Aapo L. Aro ${ }^{\mathrm{a}, \mathrm{b}}$, Sumeet S. Chugh ${ }^{\mathrm{a}, *}$ \\ a Heart Institute, Cedars-Sinai Medical Center, Los Angeles, CA, United States \\ ${ }^{\mathrm{b}}$ Heart and Lung Center, Helsinki University Hospital, Helsinki, Finland
}

\section{A R T I C L E I N F O}

Article history:

Received 3 March 2017

Received in revised form 10 March 2017

Accepted 10 March 2017

Available online 21 March 2017

\section{Keywords:}

Sudden cardiac arrest

Pediatric

Screening

Prevention

Defibrillator

\begin{abstract}
A B S T R A C T
In the present review, we summarize current approaches to the prevention of sudden cardiac death (SCD) in children and young adults, focusing on age $<35$ years. SCD in the young is rare, but devastating from the societal perspective. While coronary artery disease is the main etiology of SCD in the older age groups, conditions such as cardiomyopathies and electrical channelopathies are more likely to be found in the young. In the majority of younger cases, cardiac arrest can be the first recognized manifestation of the underlying cardiac pathology, although some have experienced cardiovascular symptoms prior to the SCD. Since identification of a cardiac disease is pivotal for implementation of appropriate prevention, measures such as electrocardiographic screening in subpopulations such as athletes have been proposed. However, these efforts are impeded by the large number of individuals needed to test in order to find one with cardiac disease, leading to significant rates of false positive findings and high costs. When a high-risk cardiac condition is identified in a young person, measures of lifestyle modification, appropriate medical treatment and ICD implantation in selected individuals based on risk stratification are warranted. Nevertheless, the benefits of lifelong ICD therapy need to be balanced with long-term complications and quality of life.
\end{abstract}

(c) 2017 Elsevier B.V. All rights reserved.

\section{Magnitude of the problem}

Sudden cardiac death (SCD) in a young person is a rare but devastating event for the family and also for the community. The estimated incidence of pediatric and young adult SCD has varied in the published literature due to differences in age range as well as in methods for SCD adjudication. If children of age less than one year are excluded [1, 2 ], recent estimates of SCD incidence for age 1 to 35 years have ranged from $0.8-2.8$ per 100,000 person-years [3-5]. However, the actual burden of SCD is disproportionately larger among the young, due to significantly more years of potential life lost in this population [6]. The incidence of SCD in children and young adults also varies depending on age and sex. At age one to four, children have a higher incidence of SCD than those aged 5-10 years who represent the age group at lowest risk. After 15 years of age the incidence of SCD starts to progressively increase, with those aged 31-35 years having up to 10 times higher incidence than age 1-10 years [7]. Epidemiological studies have also consistently demonstrated that SCD is significantly more prevalent

is Funded by National Institutes of Health, National Heart Lung and Blood Institute (NHLBI) grants R01HL122492 and R01HL126938 to Dr Chugh. Dr Chugh holds the Pauline and Harold Price Chair in Cardiac Electrophysiology at Cedars-Sinai, Los Angeles, CA, USA.

㶦败 Authors declare there is no conflict of interest.

* Corresponding author at: Cedars-Sinai Medical Center, Heart Institute, Advanced Health Sciences Pavilion, Suite A3100, 127 S. San Vicente Blvd., Los Angeles, CA 90048, United States.

E-mail address: sumeet.chugh@cshs.org (S.S. Chugh). among males compared to females, by a factor of approximately 2:1 (3-5).

\section{Etiologies of SCD in the young}

The cardiac abnormalities underlying SCD in children and adolescents include congenital heart disease, coronary artery anomalies, myocarditis, hypertrophic cardiomyopathy (HCM), dilated cardiomyopathy, left ventricular non-compaction cardiomyopathy, arrhythmogenic right ventricular cardiomyopathy (ARVC) and ion channelopathies such as long QT syndrome (LQTS), catecholaminergic polymorphic ventricular tachycardia (CPVT) and Brugada syndrome [3-5,8-10]. The estimated prevalence rates of these conditions vary widely in the general population. For example, overall prevalence of HCM is estimated to be 1 per 500 [11] and LQTS around 1 per 2500 persons [12]. The etiologies of SCD are different depending on the age group. In the majority of children aged 1-4 years (if those with severe congenital heart disease are excluded), the heart is structurally normal and SCD is presumably due to primary electrical disorders. In contrast, structural heart disease begins to dominate in older children and young adults $[4,8]$. Coronary artery disease (CAD) accounts for the great majority of SCD burden in middle-aged and elderly patients [13], but premature CAD is also the major cause of SCD in young adults aged over 25 years [8]. In a pooled clinico-pathological series in the general population $<40$ years old, premature CAD was responsible for $31 \%$, myocarditis for $9 \%$, and left ventricular hypertrophy and hypertrophic cardiomyopathy (HCM) each 
for $8 \%$ of SCD [10]. In up to $30-40 \%$ of cases, the autopsy does not reveal any cardiac structural abnormalities $[3,14,15]$, pointing to a primary electrical disorder such as LQTS, CPVT or Brugada syndrome $[5,9,16]$.

\section{Strategies for SCD prevention}

Since CAD accounts for the great majority of SCA cases in the adult population, modification of CAD risk factors and treatment of acute and chronic manifestations also impacts SCD burden at the population level [17]. Moreover, among high-risk subjects such as those with severely reduced left ventricular ejection fraction (LVEF), optimal medical heart failure therapy together with implantable cardioverter-defibrillators (ICDs) in selected high-risk patients has demonstrated clear survival benefit [18]. However, among under age 35 years and especially among children and adolescents, these approaches to SCD prevention have only limited relevance due to the significantly different spectrum of underlying etiologies. Advances in emergency medical care are an important part of improving survival from cardiac arrest, and efforts to improve measures such as bystander cardiopulmonary resuscitation (CPR) and early defibrillation are likely to improve outcomes [19,20]. However, even with ongoing advances in resuscitation, survival rates from SCA have plateaued, demanding other, more pre-emptive approaches to prevent SCD in the general population [21].

\subsection{Prevention of coronary artery disease}

The atherosclerotic process begins early in life, and the presence of cardiovascular risk factors in childhood and adolescence are associated with worse cardiometabolic health in adulthood [22-24]. This strongly suggests that pursuit of optimal cardiovascular health in childhood, i.e. primordial prevention, is important in preventing cardiovascular events in adulthood. In particular, identification and treatment of individuals at high risk of developing premature $\mathrm{CAD}$, such as those with familial hypercholesterolemia [25], is likely to reduce CAD-related SCD burden in the young adult population where premature CAD is a major cause of $\mathrm{SCD}[8]$.

\subsection{General cardiac screening}

Since cardiac arrest is often the first recognized symptom of an underlying cardiac condition in the young, there has been a longstanding interest in the concept of cardiovascular screening. The intent would be to prospectively identify or raise suspicion of a previously unrecognized cardiac disease capable of causing SCD in a previously asymptomatic subject. For the affected individuals identified with screening, there would thus be a window of opportunity for interventions aimed at preventing SCD. Among the possible preventive steps would be avoidance of strenuous physical activity or disqualification from competitive sports, appropriate medical therapy for the cardiac disease (such as beta blockers in LQTS and CPVT) and primary prevention ICD therapy for selected patients at highest risk. Several different screening programs have been proposed for different populations, or for subgroups such as athletes considered to be at high risk of SCD. In fact, pre-participation screening of athletes for underlying cardiovascular disease is either mandatory or under discussion in several countries [26]. However, most cardiac arrests occur at rest or during light activity [5], and definitive evidence for the benefits compared to costs and potential harm of these approaches to SCD preventions is still lacking [26]. History and physical examination alone are relatively ineffective in identifying subjects at elevated risk [27], so using a standard 12-lead electrocardiogram (ECG) based screening to detect underlying cardiac pathology has been a major focus of potential screening strategies [28]. The resting 12-lead ECG is reasonably sensitive in diagnosing several of the SCD etiologies such as HCM, LQTS, Brugada syndrome, ARVC and Wolff-ParkinsonWhite syndrome, but the positive predictive value of ECG for true cardiac pathology is generally low, leading to a need for screening substantial numbers to effectively diagnose or exclude underlying cardiovascular disease $[26,28]$. For example, anterior T-wave inversions in the right precordial leads are a characteristic finding in ARVC, but in the general population this ECG phenomenon carries a benign prognosis and is rarely associated with cardiac pathology [29,30].

The number of abnormal ECG findings identified via screening depends on the diagnostic criteria utilized, as well as the population of interest. Children and adolescents are particularly challenging in this respect due to alterations in electrocardiographic depolarization and repolarization patterns during aging and growth. Race and sex affect ECG as well, and certain ECG patterns such as anterior T-wave inversions are much more common in black athletes compared to whites [31]. According to a recent study, ECGs of up to one fifth of young non-athletes and a third of athletes were judged to be pathological on the bases of the criteria presented in a 2010 European Society of Cardiology document $[32,33]$. Due to the aforementioned challenges, the cost of ECG screening in pediatric or young populations are relatively high compared to potential benefits [34], and systematic mass screenings of athletes or young people from the general population are not generally recommended [26,35].

\subsection{Screening of relatives}

In contrast to broad screening efforts aimed at a large segment of the general population, screening of the family members of an individual with potentially hereditable cardiac disease represents a more focused and widely accepted way of identifying those with a high likelihood of having cardiac pathology [36]. In many institutions, testing of family members is part of routine clinical practice for the relatives of patients with potentially inheritable cardiac disease. This approach is a relatively effective approach for identifying affected individuals. For example, a study from New Zealand demonstrated that using a national registry for LQTS, an average of 2 affected family members could be identified per proband [37]. In the case of a SCD event in the young, identification of affected family members depends largely on detection or exclusion of a structural cardiac disease at the autopsy. In the absence of structural abnormalities, genetic testing of the deceased, so called molecular autopsy, may be helpful in identifying an inheritable genetic disease in the diseased. This can subsequently lead to testing of the surviving family members and enables initiation of appropriate therapy in the affected relatives [9]. In general, molecular autopsy has revealed a clinically relevant cardiac gene mutation such as mutation associated with LQTS or CPVT in approximately one-third of subjects with unexplained SCD $[5,38]$. If a specific cardiac diagnosis is determined for the deceased, comprehensive clinical assessment of families with SUD inheritable heart disease may lead to a diagnosis in $18-53 \%$ of cases, long QT syndrome being the most common finding $[9,39,40]$.

\subsection{Warning symptoms}

Another group that may benefit from aggressive diagnostic approach are those who present with cardiac symptoms prior to their SCD. Aborted cardiac arrest represents a symptom that is highly predictive of future SCD, and it is presently a well-recognized indication for ICD therapy [41]. In addition, several studies have demonstrated that most children and young adults with SCD have experienced at least some cardiovascular symptoms prior to the cardiac arrest [42-45]. Symptoms such as chest pain, palpitations and dyspnea are non-specific, but many experience potentially serious symptoms; for example, over one fourth of cardiac arrest victims have reported syncope or pre-syncope prior to SCD $[42,43]$. In many cases, these symptoms are brought to the attention of health care providers, but may not have been recognized as warning signs of an underlying life-threatening cardiac disease [42]. However, if prodromal symptoms preceding cardiac arrest lead to a 911 call, data from Oregon Sudden Unexpected Death study has demonstrated in an adult population that survival is over 5- 

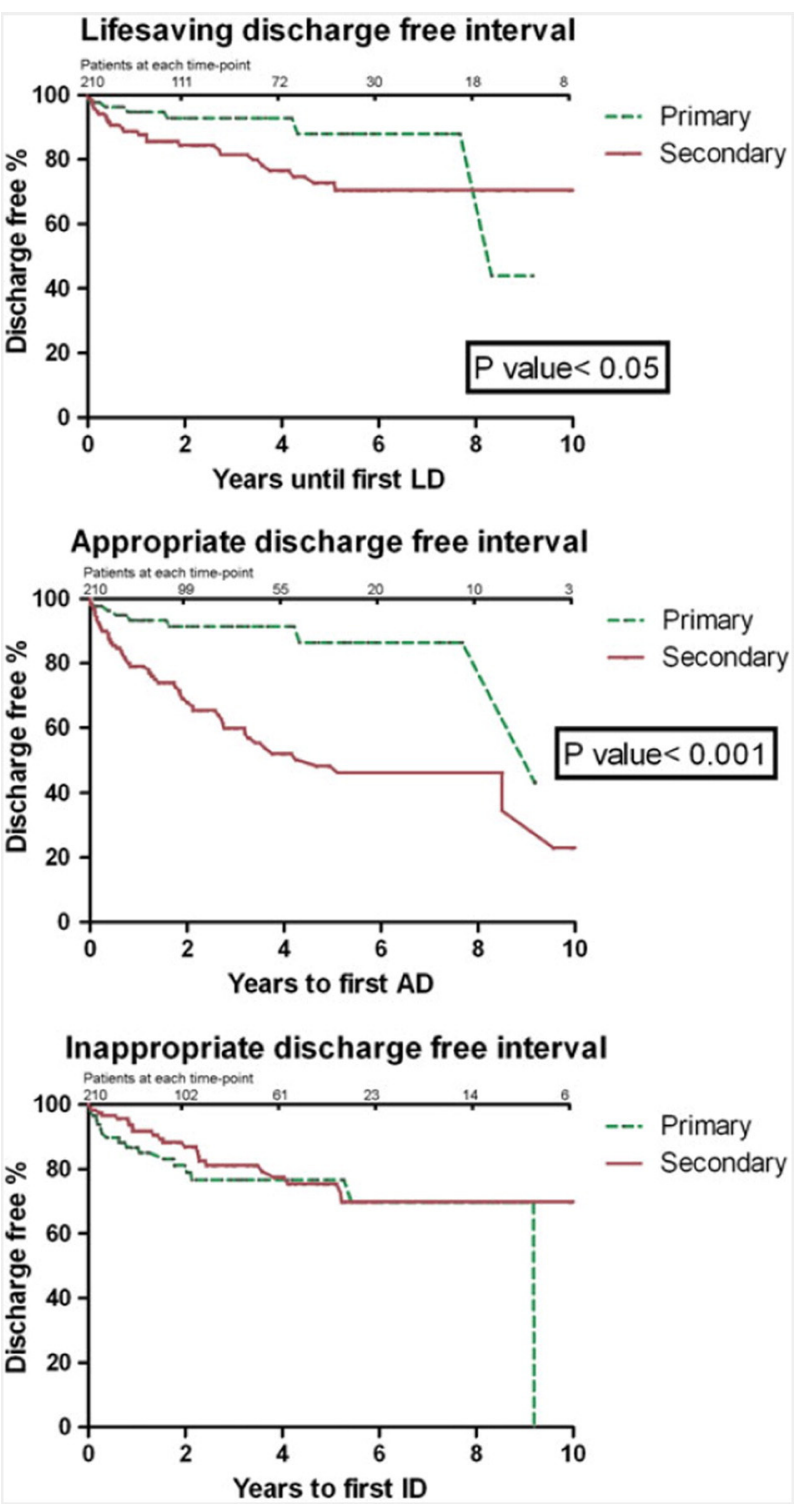

Fig. 1. Outcomes of children and young adults age $<30$ years, after implantation of primary prevention vs secondary prevention ICD 1992-2007 [55]. Re-printed with permission.

fold higher among those who requested emergency medical services compared to those who did not call 911 [46].

Especially in children, symptoms can often be vague and difficult to pinpoint, and optimal diagnostic approaches in such patients have yet to be determined. However, detailed clinical investigation including a 12-lead ECG is probably warranted for most young patients with suspected cardiovascular symptoms. The ECG is a relatively sensitive method for identifying individuals with abnormalities for a further evaluation; for example, over $80 \%$ of SCD patients with a final diagnosis of ARVC or HCM have had abnormal ECG findings prior to the SCD [47]. The positive predictive value of abnormal ECG patterns is likely to increase in subgroups with higher prevalence of cardiac disease (as is expected among those with cardiovascular symptoms) compared to the general population, making it more effective than unselected screening. However, this approach would require wide efforts to educate general practitioners and family doctors on the importance of obtaining the ECG in the appropriate patient. Also subtle ECG changes may be difficult to detect, although automated analyses generated by diagnostic algorithms of modern ECG machines are likely to be increasingly useful tools for this purpose [48]. For some conditions that increase risk of SCD, for example congenital coronary artery anomalies, the 12-lead
ECG, stress ECG and echocardiography are unlikely to be abnormal [49] and additional imaging such as cardiac CT and MRI are needed to make a diagnosis and perform corrective surgery [50]. Furthermore, focusing on the population with likely cardiac symptoms will miss the significant number of young SCD patients who don't complain of their symptoms or seek medical attention prior to the arrest. Therefore, further research is needed in order to identify the optimal combination of clinical and ECG risk markers for recognizing patients at imminent risk of SCD.

\subsection{Bystander CPR and early defibrillation}

In the event of out-of-hospital cardiac arrest, bystander CPR and early access to automated external defibrillators (AEDs) can affect survival outcomes. There is emerging evidence that the ongoing attempts to increase bystander CPR together with wider implementation of public access defibrillator programs are likely to significantly improve cardiac arrest survival among the young [19,20,51,52]. Targeted CPR education for family members of subjects at high risk of SCD is a reasonable approach and could potentially improve outcomes, although definitive evidence for the benefits of this approach is still awaited [53].

\section{Risk stratification and primary prevention ICD therapy in established cardiac disease}

Identification of a cardiac disease substrate, whether in relatives of the proband or by cardiac screening of a population sub-group, is the key to improved prevention [14]. However, only a small minority of young SCD patients have a cardiovascular disease diagnosed prior to their cardiac arrest. For example, in a Danish study of children age 118 only $18 \%$ of all SCD cases had cardiac abnormalities, mostly congenital cardiac disease, diagnosed during their lifetime [45]. When a highrisk cardiac diagnosis is established, specific preventive measures, including ICD therapy in selected patients, can be undertaken. Utilization of ICDs for primary prevention of SCD in children has been increasing and is roughly equivalent to the proportion of secondary prevention ICDs, but overall, children still account for only a very small percentage of overall ICD recipients [41]. The largest sub-group of young patients with ICDs are those with primary electrical cardiac disease (approximately $40 \%$ ), followed by those with cardiomyopathies and congenital heart disease (approximately 30\% each) [54,55]. There are few randomized trials conducted among pediatric patients and subjects with congenital heart disease, but retrospective analyses have demonstrated the efficacy of ICD therapy in children, with an approximately $25 \%$ of patients receiving appropriate shocks from the device [56]. Substantially higher rates of appropriate therapy are observed in patients implanted

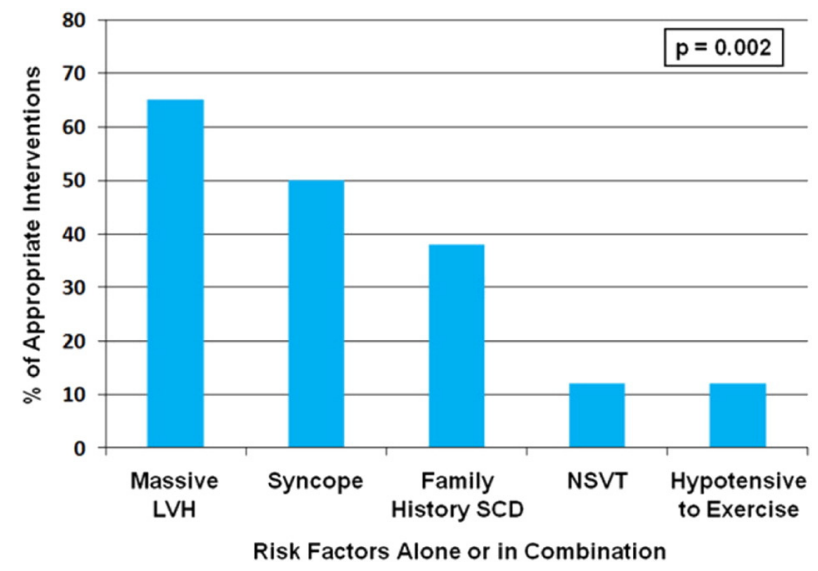

Fig. 2. Distribution of primary prevention risk factors in age $<20$-year-old hypertrophic cardiomyopathy patients with an appropriate ICD intervention [57]. Re-printed with permission. 


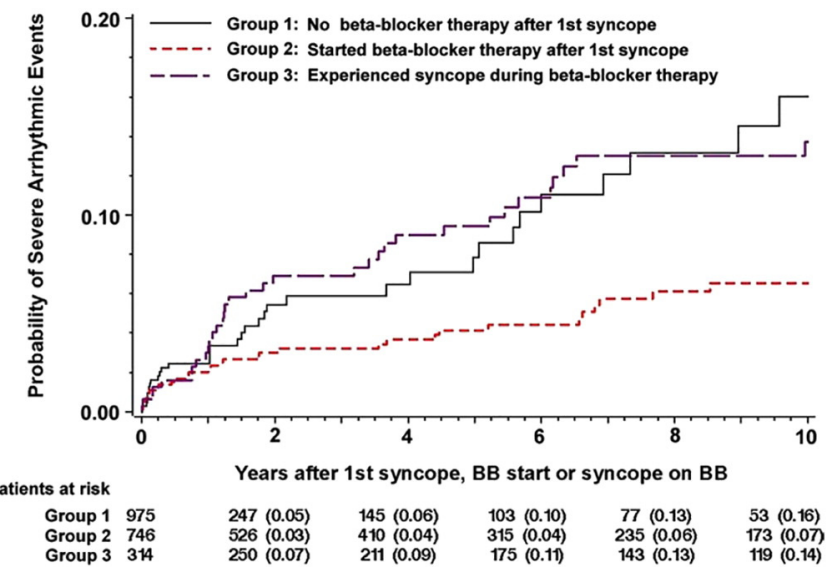

Fig. 3. Risk of severe arrhythmic events after first syncope in LQTS according to betablocker therapy status [61]. Re-printed with permission.

for secondary prevention indications compared to primary prevention [55,57] (Fig. 1).

The recommendations for use of ICDs are generally not age-specific, so for pediatric patients the guidelines are similar to adults. ICD therapy for secondary prevention is indicated for survivors of cardiac arrest or hemodynamically unstable ventricular tachycardia (VT) without clearly reversible cause such as Wolff-Parkinson-White syndrome with pre-excited atrial fibrillation, or myocarditis [58]. Those with structural heart disease who experience sustained hemodynamically stable VT should also generally receive an ICD for secondary prevention. As for adults, primary prevention ICD therapy is generally recommended for young patients with severely reduced LVEF $<30-35 \%$ [41].

In addition, for some conditions such as HCM, ARVC, LQTS, CPVT and Brugada syndrome, specific guidelines for SCD preventive measures such as lifestyle modifications and use of medications and device therapy exist and can be followed also in pediatric and adolescent patients [41,58-60]. In HCM, prior cardiac arrest or sustained VT, extreme left ventricular hypertrophy, syncope, family history of HCM-related SCD and abnormal blood pressure response to exercise or nonsustained VTs (when manifesting together with other risk modifiers) are risk factors for cardiac arrest that warrant consideration of a primary prevention ICD also for pediatric patients [57,59] (Fig. 2). In LQTS patients, in addition to lifestyle changes such as avoidance of QTprolonging drugs and arrhythmia triggers, beta-blockers are the mainstay of prevention for those with a history of syncope and for those having significantly prolonged QTc interval [61] (Fig. 3). In LQTS, ICD therapy should be reserved only for those who experience recurrent syncopal episodes or VT while on beta-blockers [60]. Similarly, for CPVT patients, implantation of an ICD is recommended only for those who have syncopal episodes or bidirectional/polymorphic VT while on beta-blocker therapy [60]. In Brugada syndrome, besides cardiac arrest survivors and those with sustained VT, patients with a history of syncope judged to be caused by ventricular arrhythmias are likely to benefit from ICD implantation [60].

\subsection{Additional considerations regarding ICD therapy}

The impact of receiving an ICD may be considerably greater for a young person than for older individuals, highlighting the need to carefully balance the risks and adverse effects of these devices with potential survival benefit. Many of the youngest patients outgrow the length of their intra-cardiac leads and outlive the functionality of their devices. As a consequence, a large subgroup will manifest with long-term problems related to the ICD, requiring not just generator changes but also lead replacements, revisions or extractions. Inappropriate device therapy is not uncommon among the young (Fig. 1), and is usually a significant adverse event for patients, with major psychological impact and clinical implications. Data from several US pediatric centers demonstrated that inappropriate shocks occur in approximately $20 \%$ of

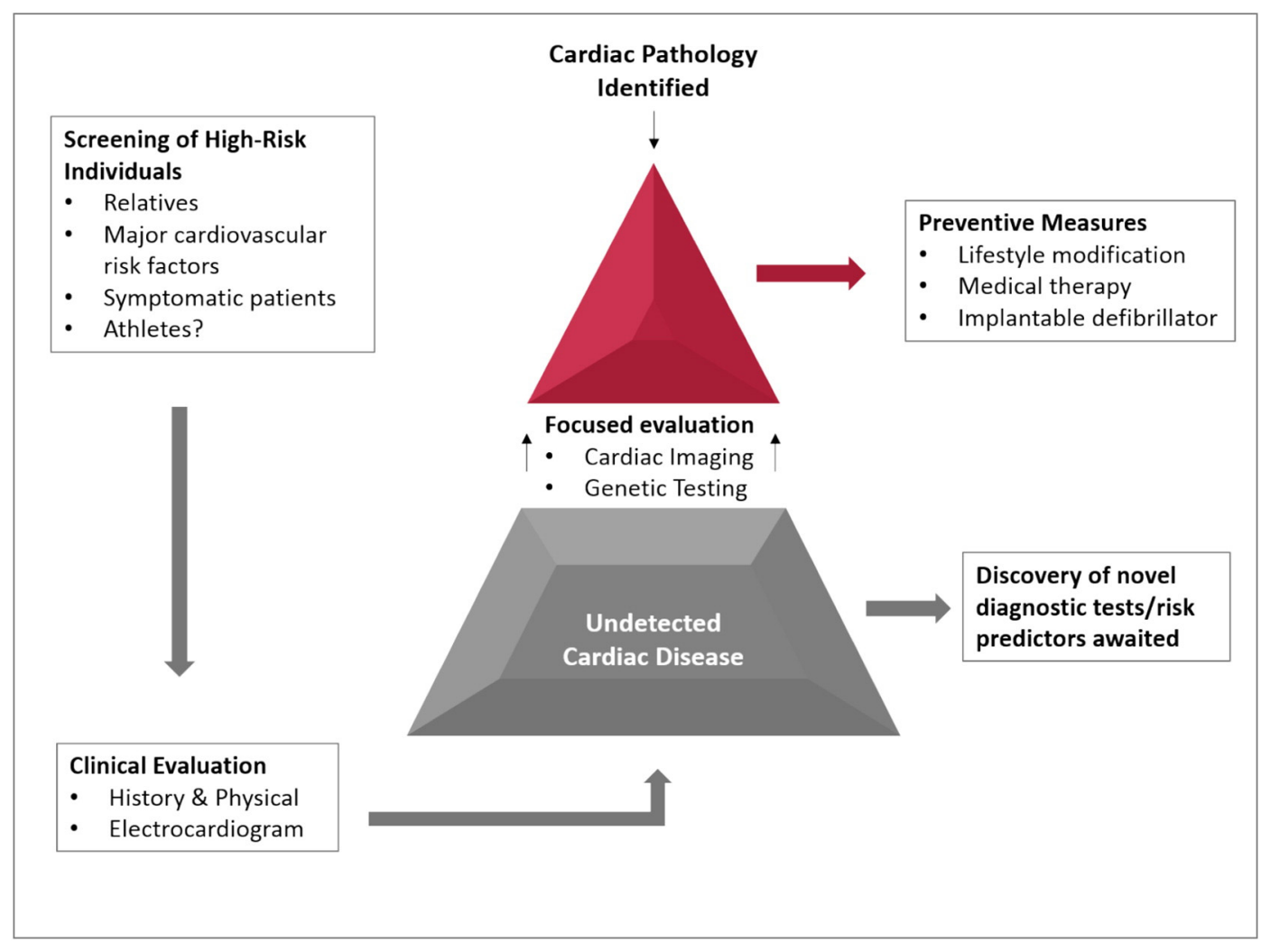

Fig. 4. Current and future approaches to sudden cardiac arrest prevention in the young. 
patients with an ICD, with a mean of six inappropriate shocks per patient [56]. Most of the inappropriate therapies are attributable to lead failure, followed by supraventricular tachycardias and over-sensing $[56,62,63]$. Consequently, the subcutaneous ICD has recently emerged as an appealing alternative to the intra-cardiac device for a carefully selected subgroup of patients [64]. In addition, for some patients at potentially high risk of SCD but not meeting definite indications for implantation of a permanent ICD, the wearable cardioverter-defibrillator can be a short-term solution $[65,66]$.

\section{Conclusions}

Prevention of SCD in the young remains a significant challenge, and widespread screening efforts for underlying causes are unlikely to be feasible due the low prevalence of this tragic event. Targeted screening for subgroups at highest risk, such as relatives of SCD victims or of those with hereditable cardiac disease capable of causing SCD, in addition to individuals with cardiac symptoms, are likely to provide the highest yield. However, this approach fails to identify a large proportion of young SCD patients, so novel risk markers and tools are needed in order to better recognize asymptomatic subjects with underlying potentially life-threatening cardiac pathology (Fig. 4). If a cardiac diagnosis can be established, optimal preventive measures such as lifestyle adaptation, medical therapy and ICD implantation for those at highest risk should be implemented. However, ICD therapy needs to be balanced with potential long-term adverse effects and complications, and further research is needed to more clearly identify those who are most likely to benefit from an ICD.

\section{References}

[1] Chugh SS, Reinier K, Balaji S, et al. Population-based analysis of sudden death in children: the Oregon Sudden Unexpected Death Study. Heart Rhythm 2009;6:1618-22.

[2] Moon RY, Horne RS, Hauck FR. Sudden infant death syndrome. Lancet 2007;370: 1578-87.

[3] Winkel BG, Holst AG, Theilade J, et al. Nationwide study of sudden cardiac death in persons aged 1-35 years. Eur Heart J 2011:32:983-90.

[4] Pilmer CM, Kirsh JA, Hildebrandt D, Krahn AD, Gow RM. Sudden cardiac death in children and adolescents between 1 and 19 years of age. Heart Rhythm 2014;11: 239-45.

[5] Bagnall RD, Weintraub RG, Ingles J, et al. A prospective study of sudden cardiac death among children and young adults. N Engl J Med 2016;374:2441-52.

[6] Stecker EC, Reinier K, Marijon E, et al. Public health burden of sudden cardiac death in the United States. Circ Arrhythm Electrophysiol 2014:7:212-7.

[7] Chugh SS, Jui J, Gunson K, et al. Current burden of sudden cardiac death: multiple source surveillance versus retrospective death certificate-based review in a large U.S. community. J Am Coll Cardiol 2004;44:1268-75.

[8] Meyer L, Stubbs B, Fahrenbruch C, et al. Incidence, causes, and survival trends from cardiovascular-related sudden cardiac arrest in children and young adults 0 to 35 years of age: a 30-year review. Circulation 2012;126:1363-72.

[9] Semsarian C, Ingles J, Wilde AA. Sudden cardiac death in the young: the molecular autopsy and a practical approach to surviving relatives. Eur Heart J 2015;36:1290-6.

[10] van der Werf C, van Langen IM, Wilde AA. Sudden death in the young: what do we know about it and how to prevent? Circ Arrhythm Electrophysiol 2010;3:96-104.

[11] Maron BJ, Gardin JM, Flack JM, Gidding SS, Kurosaki TT, Bild DE. Prevalence of hypertrophic cardiomyopathy in a general population of young adults. Echocardiographic analysis of 4111 subjects in the CARDIA Study. Coronary Artery Risk Development in (Young) Adults. Circulation 1995:92:785-9.

[12] Schwartz PJ, Stramba-Badiale M, Crotti L, et al. Prevalence of the congenital long-QT syndrome. Circulation 2009;120:1761-7.

[13] Huikuri HV, Castellanos A, Myerburg RJ. Sudden death due to cardiac arrhythmias. N Engl J Med 2001;345:1473-82.

[14] Chugh SS. Early identification of risk factors for sudden cardiac death. Nat Rev Cardiol 2010;7:318-26

[15] Chugh SS. Approach to unexplained sudden death in the young: proactive during life and prospective at death. Europace 2011;13:1364-5.

[16] Ackerman M, Atkins DL, Triedman JK. Sudden cardiac death in the young. Circulation 2016;133:1006-26

[17] Deo R, Albert CM. Epidemiology and genetics of sudden cardiac death. Circulation 2012;125:620-37.

[18] Yousuf O, Chrispin J, Tomaselli GF, Berger RD. Clinical management and prevention of sudden cardiac death. Circ Res 2015;116:2020-40.

[19] Bardai A, Berdowski J, van der Werf C, et al. Incidence, causes, and outcomes of outof-hospital cardiac arrest in children. A comprehensive, prospective, populationbased study in the Netherlands. J Am Coll Cardiol 2011;57:1822-8.

[20] Chugh SS. Improved outcomes for cardiac arrest in children. Share the baton with the bystander. J Am Coll Cardiol 2011;57:1829-30.
[21] Naim MY, Burke RV, McNally BF, et al. Association of bystander cardiopulmonary resuscitation with overall and neurologically favorable survival after pediatric out-ofhospital cardiac arrest in the United States: a report from the Cardiac Arrest Registry to Enhance Survival Surveillance Registry. JAMA Pediatr 2016.

[22] Berenson GS. Childhood risk factors predict adult risk associated with subclinical cardiovascular disease. The Bogalusa Heart Study. Am J Cardiol 2002;90:3L-7L.

[23] Laitinen TT, Pahkala K, Magnussen CG, et al. Ideal cardiovascular health in childhood and cardiometabolic outcomes in adulthood: the Cardiovascular Risk in Young Finns Study. Circulation 2012;125:1971-8.

[24] Hartiala O, Magnussen CG, Kajander S, et al. Adolescence risk factors are predictive of coronary artery calcification at middle age: the cardiovascular risk in young Finns study. J Am Coll Cardiol 2012;60:1364-70.

[25] Knowles JW, O'Brien EC, Greendale K, et al. Reducing the burden of disease and death from familial hypercholesterolemia: a call to action. Am Heart J 2014;168: 807-11.

[26] Maron BJ, Friedman RA, Kligfield P, et al. Assessment of the 12-lead electrocardiogram as a screening test for detection of cardiovascular disease in healthy general populations of young people (12-25 years of age): a scientific statement from the American Heart Association and the American College of Cardiology. J Am Coll Cardiol 2014;64:1479-514.

[27] Anderson JB, Grenier M, Edwards NM, et al. Usefulness of combined history, physical examination, electrocardiogram, and limited echocardiogram in screening adolescent athletes for risk for sudden cardiac death. Am J Cardiol 2014;114:1763-7.

[28] Rodday AM, Triedman JK, Alexander ME, et al. Electrocardiogram screening for disorders that cause sudden cardiac death in asymptomatic children: a meta-analysis. Pediatrics 2012;129:e999-1010.

[29] Aro AL, Anttonen O, Tikkanen JT, et al. Prevalence and prognostic significance of Twave inversions in right precordial leads of a 12-lead electrocardiogram in the middle-aged subjects. Circulation 2012:125:2572-7.

[30] Malhotra A, Dhutia H, Gati S, et al. Anterior T-wave inversion in young White athletes and nonathletes. J Am Coll Cardiol 2017;69:1-9.

[31] Papadakis M, Carre F, Kervio G, et al. The prevalence, distribution, and clinical outcomes of electrocardiographic repolarization patterns in male athletes of African/ Afro-Caribbean origin. Eur Heart J 2011;32:2304-13.

[32] Corrado D, Pelliccia A, Heidbuchel H, et al. Recommendations for interpretation of 12-lead electrocardiogram in the athlete. Eur Heart J 2010;31:243-59.

[33] Chandra N, Bastiaenen R, Papadakis M, et al. Prevalence of electrocardiographic anomalies in young individuals: relevance to a nationwide cardiac screening program. J Am Coll Cardiol 2014;63:2028-34.

[34] Leslie LK, Cohen JT, Newburger JW, et al. Costs and benefits of targeted screening for causes of sudden cardiac death in children and adolescents. Circulation 2012;125: 2621-9.

[35] Kaltman JR, Thompson PD, Lantos J, et al. Screening for sudden cardiac death in the young: report from a national heart, lung, and blood institute working group. Circulation 2011;123:1911-8.

[36] Ranthe MF, Winkel BG, Andersen EW, et al. Risk of cardiovascular disease in family members of young sudden cardiac death victims. Eur Heart J 2013;34:503-11.

[37] Earle N, Crawford J, Smith W, et al. Community detection of long QT syndrome with a clinical registry: an alternative to ECG screening programs? Heart Rhythm 2013; 10:233-8.

[38] Tester DJ, Ackerman MJ. Postmortem long QT syndrome genetic testing for sudden unexplained death in the young. J Am Coll Cardiol 2007;49:240-6.

[39] Behr ER, Dalageorgou C, Christiansen M, et al. Sudden arrhythmic death syndrome: familial evaluation identifies inheritable heart disease in the majority of families. Eur Heart J 2008;29:1670-80.

[40] Kumar S, Peters S, Thompson T, et al. Familial cardiological and targeted genetic evaluation: low yield in sudden unexplained death and high yield in unexplained cardiac arrest syndromes. Heart Rhythm 2013;10:1653-60.

[41] Epstein AE, DiMarco JP, Ellenbogen KA, et al. 2012 ACCF/AHA/HRS focused update incorporated into the ACCF/AHA/HRS 2008 guidelines for device-based therapy of cardiac rhythm abnormalities: a report of the American College of Cardiology Foundation/American Heart Association Task Force on Practice Guidelines and the Heart Rhythm Society. Circulation 2013;127:e283-352.

[42] Drezner JA, Fudge J, Harmon KG, Berger S, Campbell RM, Vetter VL. Warning symptoms and family history in children and young adults with sudden cardiac arrest. J Am Board Fam Med 2012;25:408-15.

[43] Wisten A, Messner T. Symptoms preceding sudden cardiac death in the young are common but often misinterpreted. Scand Cardiovasc J 2005;39:143-9.

[44] Liberthson RR. Sudden death from cardiac causes in children and young adults. N Engl J Med 1996;334:1039-44.

[45] Winkel BG, Risgaard B, Sadjadieh G, Bundgaard H, Haunso S, Tfelt-Hansen J. Sudden cardiac death in children (1-18 years): symptoms and causes of death in a nationwide setting. Eur Heart J 2014;35:868-75.

[46] Marijon E, Uy-Evanado A, Dumas F, et al. Warning symptoms are associated with survival from sudden cardiac arrest. Ann Intern Med 2016;164:23-9.

[47] Wisten A, Andersson S, Forsberg H, Krantz P, Messner T. Sudden cardiac death in the young in Sweden: electrocardiogram in relation to forensic diagnosis. J Intern Med 2004:255:213-20.

[48] Guglin ME, Thatai D. Common errors in computer electrocardiogram interpretation. Int J Cardiol 2006;106:232-7.

[49] Basso C, Maron BJ, Corrado D, Thiene G. Clinical profile of congenital coronary artery anomalies with origin from the wrong aortic sinus leading to sudden death in young competitive athletes. J Am Coll Cardiol 2000;35:1493-501.

[50] Brothers JA, Whitehead KK, Keller MS, et al. Cardiac MRI and CT: differentiation of normal ostium and intraseptal course from slitlike ostium and interarterial course in anomalous left coronary artery in children. Am J Roentgenol 2015;204:W104-9. 
[51] Vetter VL, Haley DM. Secondary prevention of sudden cardiac death: does it work in children? Curr Opin Cardiol 2014;29:68-75

[52] Iwami T, Kitamura T, Kiyohara K, Kawamura T. Dissemination of chest compressiononly cardiopulmonary resuscitation and survival after out-of-hospital cardiac arrest. Circulation 2015;132:415-22.

[53] Cartledge S, Bray JE, Leary M, Stub D, Finn J. A systematic review of basic life support training targeted to family members of high-risk cardiac patients. Resuscitation 2016;105:70-8.

[54] Burns KM, Evans F, Kaltman JR. Pediatric ICD utilization in the United States from 1997 to 2006. Heart Rhythm 2011;8:23-8.

[55] Von Bergen NH, Atkins DL, Dick 2nd M, et al. Multicenter study of the effectiveness of implantable cardioverter defibrillators in children and young adults with heart disease. Pediatr Cardiol 2011;32:399-405.

[56] Berul CI, Van Hare GF, Kertesz NJ, et al. Results of a multicenter retrospective implantable cardioverter-defibrillator registry of pediatric and congenital heart disease patients. J Am Coll Cardiol 2008;51:1685-91.

[57] Maron BJ, Spirito P, Ackerman MJ, et al. Prevention of sudden cardiac death with implantable cardioverter-defibrillators in children and adolescents with hypertrophic cardiomyopathy. J Am Coll Cardiol 2013;61:1527-35.

[58] Berul CI. Implantable cardioverter defibrillator criteria for primary and secondary prevention of pediatric sudden cardiac death. Pacing Clin Electrophysiol 2009; 32(Suppl. 2):S67-70.

[59] Gersh BJ, Maron BJ, Bonow RO et al. 2011 ACCF/AHA guideline for the diagnosis and treatment of hypertrophic cardiomyopathy: a report of the American College of Cardiology Foundation/American Heart Association Task Force on Practice Guidelines. Developed in collaboration with the American Association for Thoracic Surgery, American Society of Echocardiography, American Society of Nuclear Cardiology,
Heart Failure Society of America, Heart Rhythm Society, Society for Cardiovascular Angiography and Interventions, and Society of Thoracic Surgeons. J Am Coll Cardiol 2011;58:e212-60

[60] Priori SG, Wilde AA, Horie M, et al. HRS/EHRA/APHRS expert consensus statement on the diagnosis and management of patients with inherited primary arrhythmia syndromes: document endorsed by HRS, EHRA, and APHRS in May 2013 and by ACCF, AHA, PACES, and AEPC in June 2013. Heart Rhythm 2013;10:1932-63.

[61] Jons C, Moss AJ, Goldenberg I, et al. Risk of fatal arrhythmic events in long QT syndrome patients after syncope. J Am Coll Cardiol 2010;55:783-8.

[62] Atallah J, Erickson CC, Cecchin F, et al. Multi-institutional study of implantable defibrillator lead performance in children and young adults: results of the Pediatric Lead Extractability and Survival Evaluation (PLEASE) study. Circulation 2013;127: 2393-402.

[63] DeWitt ES, Triedman JK, Cecchin F, et al. Time dependence of risks and benefits in pediatric primary prevention implantable cardioverter-defibrillator therapy. Circ Arrhythm Electrophysiol 2014;7:1057-63.

[64] Bordachar P, Marquie C, Pospiech T, et al. Subcutaneous implantable cardioverter defibrillators in children, young adults and patients with congenital heart disease. Int J Cardiol 2016;203:251-8

[65] Klein HU, Goldenberg I, Moss AJ. Risk stratification for implantable cardioverter defibrillator therapy: the role of the wearable cardioverter-defibrillator. Eur Heart J 2013;34:2230-42

[66] Kutyifa V, Moss AJ, Klein H, et al. Use of the wearable cardioverter defibrillator in high-risk cardiac patients: data from the Prospective Registry of Patients Using the Wearable Cardioverter Defibrillator (WEARIT-II Registry). Circulation 2015;132: 1613-9. 